\title{
A rare case of bovine tuberculosis caused by Mycobacterium bovis in a domestic rabbit
}

\author{
Su Gwon Roh ${ }^{1}$, Yun-Ho Jang ${ }^{2}$, Jongho Kim ${ }^{1}$, Kyunghyun Lee ${ }^{1}$, Byungjae So ${ }^{1}$, Eun-Jin Choi ${ }^{1, *}$ \\ ${ }^{1}$ Animal Disease Diagnostic Division, Animal and Plant Quarantine Agency, Gimcheon 39660, Korea \\ ${ }^{2}$ Bacterial Disease Division, Animal and Plant Quarantine Agency, Gimcheon 39660, Korea
}

\begin{abstract}
A 12-month-old domestic rabbit died suddenly without specific clinical signs. Gross examination revealed irregular yellowish hepatic nodules with pus in the submandibular muscles, lungs, uterus, and small intestines. Histopathological examination of the liver showed granulomatous inflammation with acid-fast-positive bacteria. Mycobacterium bovis SB1040 was identified by polymerase chain reaction and spoligotyping, and Pasteurella multocida was isolated from the multiple lesions. This report demonstrates the pathological features of rare bovine tuberculosis (bTB) in a domestic rabbit, the first case in the Republic of Korea. To ensure public safety, we recommend routine monitoring of rabbits to control the incidence of bTB.
\end{abstract}

Keywords: bovine tuberculosis, granulomatous inflammation, Mycobacterium bovis, Pasteurella multocida, rabbit

\section{*Corresponding author}

\section{Eun-Jin Choi}

Animal Disease Diagnostic Division, Animal and Plant Quarantine Agency, 177 Hyeoksin 8ro, Gimcheon 39660, Korea.

Tel: +82-54-912-0460
Fax: +82-54-912-0465
E-mail: choiej@korea.kr

\section{ORCID:}

Su Gwon Roh

https://orcid.org/0000-0002-3597-6615

Yun-Ho Jang

https://orcid.org/0000-0001-8371-4399

Jongho Kim

https://orcid.org/0000-0002-3719-860X

Kyunghyun Lee

https://orcid.org/0000-0002-3113-2781

Byungjae So

https://orcid.org/0000-0002-6125-6873

Eun-Jin Choi

https://orcid.org/0000-0001-9690-7501

Conflict of Interest

The authors declare no conflicts of interest.

Received: December 12, 2019

Revise d: February 27, 2020

Accepted: March 16, 2020
Bovine tuberculosis (bTB) is a contagious and chronic zoonotic disease caused by Mycobacterium bovis. Globally, bTB is reported to occur in various hosts, such as cattle, deer, dog, cat, otter, and humans [1]. However, in the Republic of Korea, it has only been reported in cattle, deer, and otters [2]; it is transmitted via direct and indirect contact with infected animals, and its clinical signs include weakness, weight loss, intermittent hacking cough, diarrhea, and anorexia, among others [3]. Many countries have implemented preventive and control policies, such as diagnostic testing and slaughter of infected animals, vaccination, pasteurization of milk, and monitoring of wild animals, to eradicate this disease [4]. In the Republic of Korea, cattle and deer are regularly screened for bTB infection, and infected animals are culled. In addition, only bTB-negative animals are transported to farms or slaughterhouses.

Pasteurellosis, caused by Pasteurella multocida is a major bacterial disease in domestic rabbits [5]. It is transmitted primarily via direct contact, although transmission via indirect contact with infectious aerosol and with the secretions and exudates of infected vectors may also occur. P. multocida is a normal microflora in the oral cavity or upper airways of animals; however, when the host is immunocompromised due to malnutrition or stress consequent to dense breeding, it causes pathogenic infections. Pasteurellosis shows various clinical signs, such as rhinitis, pneumonia, otitis media, endometritis, orchitis, abscess, and septicemia [5].

To our knowledge, unlike pasteurellosis, bTB has rarely been reported in domestic rabbits worldwide, including the Republic of Korea. In this report, we describe the pathological findings of a rare case of bTB infection in a domestic rabbit.

On March 22, 2019, a deceased 12-month-old domestic rabbit was brought to the Animal and Plant Quarantine Agency (APQA; Korea) to identify the cause of mortality. It died suddenly without manifesting specific clinical signs, except for discharge from the eyes. The habitat was a farm breeding 100 rabbits in Yecheon, Gyeongsangbuk-do province, Republic of Korea.

Gross visualization at necropsy showed irregular yellow nodules on the liver (Fig. 1A), and multiple pus in the submandibular muscles, lungs, uterus, and small intestines. For histopathological exanimation, all organs, including the liver were fixed in $10 \%$ neutral buffered formalin for $24 \mathrm{~h}$ and were processed routinely. Finally, the formalin-fixed tissues were sectioned and stained with hematoxylin-eosin and Ziehl-Neelsen stain [2]. Histopathological exam- 

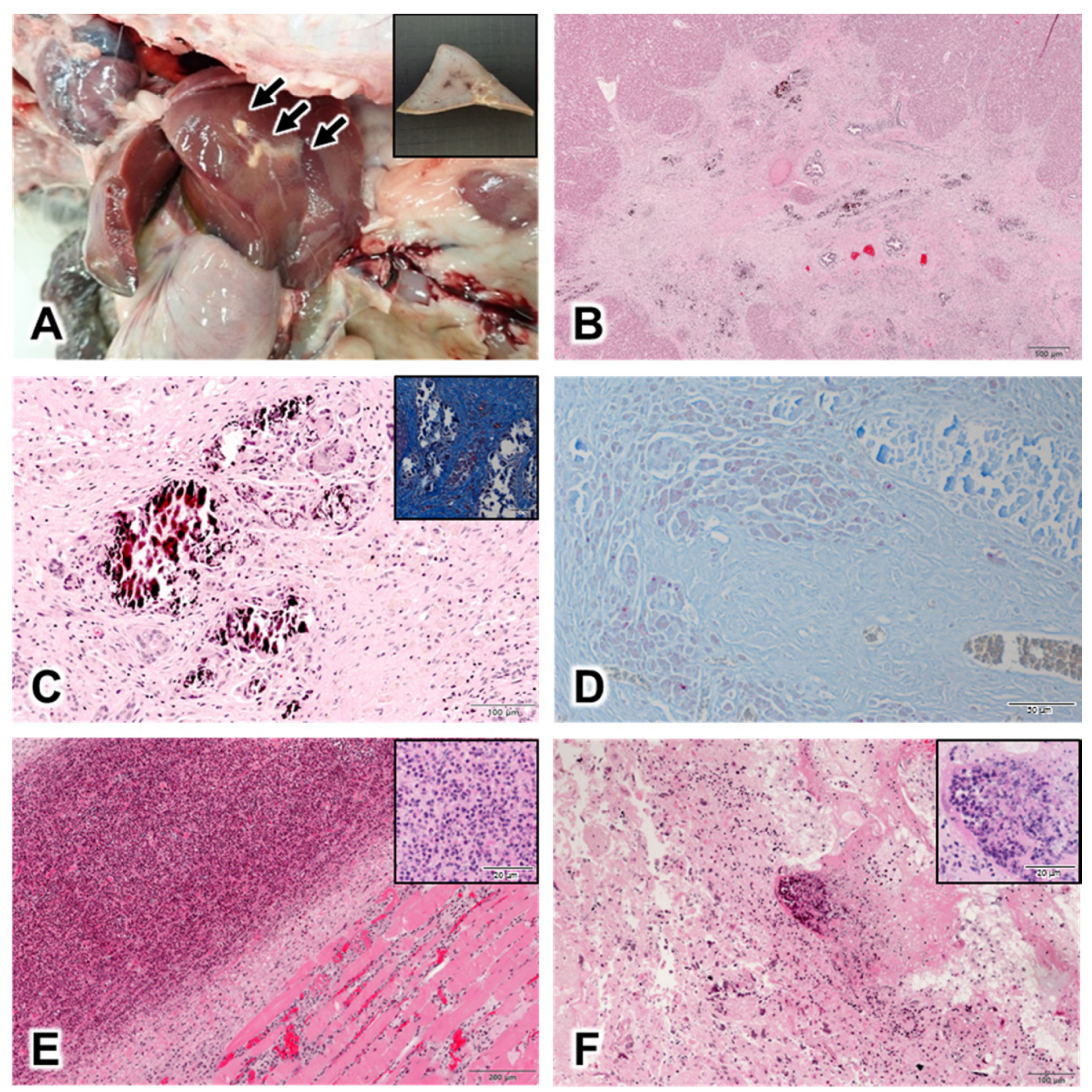

Fig. 1. Gross and histopathological findings on necropsy; (A) Liver of the rabbit. Irregular, yellow nodules are observed (arrow). Inset shows the sectioned surface of formalin-fixed liver. (B) Nodules in the liver tissue. Caseous, necrotic foci are observed on H\&E stained sections, Bar $=500 \mu \mathrm{m}$. (C) Granulomatous lesions in the liver nodule. Epithelioid macrophages, neutrophils, and Langerhans-type giant cells are observed around the region with mineralization, surrounded by proliferated connective tissues on H\&E stained sections, $\mathrm{Bar}=100 \mu \mathrm{m}$. Inset shows inflammation with fibrosis in nodule on Masson's trichrome stained sections, Bar $=100 \mu \mathrm{m}$. (D) Granuloma in the liver nodule. Pink acid-fast-positive bacteria were observed on Ziehl-Neelsen staining, Bar $=100 \mu \mathrm{m}$. (E) Suppurative inflammation under the jaw. Multiple neutrophils were observed around the skeletal muscles on H\&E stained sections Bar $=200 \mu \mathrm{m}$. Inset shows neutrophil aggregation, $B a r=50 \mu \mathrm{m}$. (F) Suppurative uteritis. Neutrophils were observed throughout the uterus on H\&E stained sections, Bar $=100 \mu \mathrm{m}$. Inset shows neutrophil accumulation, Bar $=50 \mu \mathrm{m}$. H\&E, hematoxylin \& eosin.

ination showed that most lesions were round or oval, and sometimes polymorphic in the liver. Mineralization was found in the center of granulomas, and were scattered all over the lesion. The hepatic nodules were surrounded by proliferative connective tissue indicative of granulomatous inflammation, and demonstrated mineralization; epithelioid macrophages and Langerhans-type giant cells were also observed (Fig. 1B, 1C), in addition to pink acid-fast-positive bacteria (Fig. 1D). Additionally, suppurative inflammatory lesions were present in the submandibular muscles, lungs, uterus, and small intestines; microscopically, these were composed of aggregated neutrophils, lymphocytes, and necrotic cell debris (Fig. 1E, 1F).

After gross and histopathological examination, the differential diagnosis was performed based on bacterial identification. The lesions in the tissues were inoculated in 5\% sheep blood agar (Asan Pharm. Co., Korea) and MacConkey agar (Becton Dickinson, USA), and pathogen-specific genes were amplified by polymerase chain reaction (PCR). Automated genomic DNA extraction was performed with Maxwell ${ }^{\circledR}$ RSC 48 (Promega, USA), according to the manufacturer's instructions, from homogenates of various tissues including 

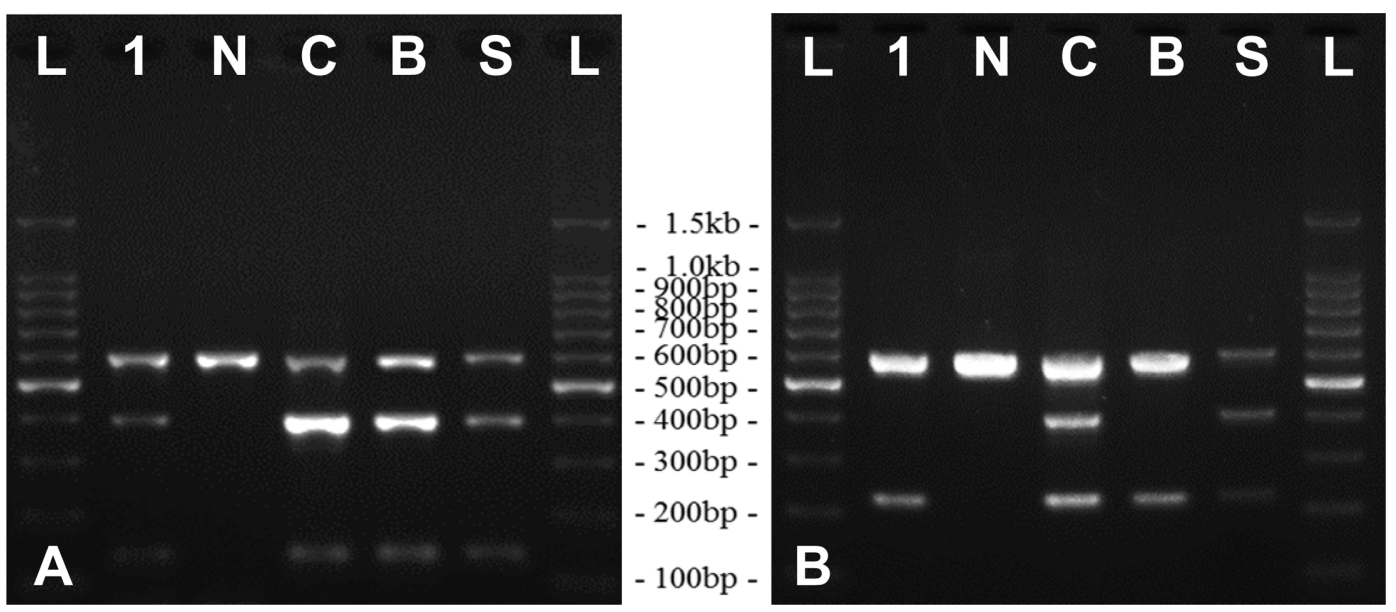

Fig. 2. Results of PCR assay for the identification of Mycobacterium spp. in the liver tissues; (A) Result of PCR assay for IS6110 and IS1081 genes of M. tuberculosis complex. The amplicon sizes are $397 \mathrm{bp}$ and $135 \mathrm{bp}$, respectively. (B) PCR result for distinguishing M. tuberculosis and M. bovis using DiaPlexC ${ }^{\mathrm{TM}} \mathrm{MTB} / M$. bovis detection kit. The amplicon size for M. tuberculosis was 406 bp and that for $M$. bovis was $223 \mathrm{bp}$. The DNA amplification band for M. bovis was detected in the liver tissue.

L, ladder; 1, sample DNA; N, negative control; C, control template; B, DNA from Mycobacterium bovis AN5; S, non-amplified standard marker; PCR, polymerase chain reaction; M. tuberculosis, Mycobacterium tuberculosis; M. bovis, Mycobacterium bovis; DNA, deoxyribose nucleic acid.

the liver, which had nodules and were suspected of tubercle. In particular, DNA extracted from the liver was used in the PCR assay for the detection of Mycobacterium tuberculosis complex, including M. tuberculosis and M. bovis. IS1081 and IS6110 regions, insertion elements in the M. tuberculosis complex genomes [6,7], were amplified using the DiaPlexC ${ }^{\mathrm{TM}}$ MTB detection kit (Solgent, Korea). PCR amplification of liver DNA was positive for specific genes of $M$. tuberculosis complex (Fig. 2A). Differentiation of M. tuberculosis and $M$. bovis DNA was performed using the DiaPlexC ${ }^{\mathrm{TM}} \mathrm{MTB} / M$. bovis detection kit (Solgent, Korea), which showed specific amplicons of M. bovis (Fig. 2B). Isolation of Mycobacterium spp. was attempted to identify the bacteria, and for further analysis, as described previously [8]. However, $M$. bovis was not isolated owing to the presence of various microbial contaminants. The differential diagnosis was performed from the suppurative foci of multiple internal organs. Suspected enriched mucoidal and non-hemolytic colonies were subjected to biochemical identification using the VITEK II system (BioMérieux, France), and bacterial identification was confirmed with species-specific kmtl amplification $[9,10]$. P. multocida was identified in the suppurative lesions.

Spacer oligonucleotide typing (Spoligotyping) was performed to determine the Mycobacterial species, and to identify its genotype. Spoligotype pattern was analyzed using the REBA MTB-Spoligotyping ${ }^{\circledR}$ kit (Optipharm M\&D, Korea), and was deposited in the $M$. bovis spoligotype database (http://www.mbovis.org) for comparison with various previously documented Mycobacterial spoligotype patterns [11,12]. The results indicated that the Mycobacterium spp. in the liver nodules was of spoligotype SB1040, previously determined in M. bovis.

Pathological features, amplification of M. tuberculosis complex-specific genes, and the spoligotype supported a diagnosis of bTB infection due to $M$. bovis. Finally, this case was diagnosed as a case of comorbid pasteurellosis and bTB.

Most bTB cases show lesions in various organs, including the respiratory tract and lymph nodes; however, in the present case, it was confined to the liver. This was considered to have occurred consequent to extrapulmonary dissemination, which is known as a particularly common transmission in rabbits infected by $M$. bovis [13]. The mycobacterial infection in this case was assumed to have been caused by the breeding environment. The rabbits were bred in burrows in bare ground, similar to wild rabbit warrens. However, as the environment has been considered to be a pathogenic reservoir [14], rabbits in this study were potentially vulnerable to infectious diseases. In this case, it was presumed that the rabbit was infected through contact with infected bodies or soil in the burrow. In addition, bovine tuberculin purified protein derivative (PPD) inoculation tests in the co-habiting rabbits from this farm were negative in all cases (data not shown). These results therefore, indicate that the possibility of air transmission is very low. However, further studies considering the epidemiological situation, is required for elucidating the path of infection.

In contrast, the pasteurellosis lesions were acute, systemic, and typical. In this case, the cause of death was assumed to be pasteurellosis caused by $P$. multocida, and not bTB by $M$. bovis. P. multocida was thought to invade acutely and systemically by an opportunistic infection into the host rabbit, which may have been immunocompromised because of chronic bTB. In addition, the cause of death in 6 co-habiting 
rabbits was identified as pasteurellosis with suppurative inflammations in their submandibular muscles, lungs, uterus, and small intestines; this further supported the spread of $P$. multocida infection.

On the basis of the Korea Animal Health Integrated System (KAHIS; http://www.kahis.go.kr), the region with mostreported bTB cases among domestic animals in the last 10 years (2010-2019) was Gyeongsangbuk-do province, which accounted for 11 of the 27 cases reported. The farm in the present report was located in this province. In addition, SB1040 has been consistently reported as a major spoligotype pattern of $M$. bovis for bTB cases in the Republic of Korea, along with SB0140, which was reported between 2012-2015 [8]. Therefore, this case was considered as a natural outbreak of bTB in the deceased rabbit. However, the correlation between the bTB outbreak and farm location was difficult to establish owing to a lack of epidemiological information.

bTB has been rarely reported in wild and domestic rabbits, although laboratory rabbits demonstrate susceptibility to experimental infection with $M$. bovis. Few isolated cases of M. bovis infection in rabbits have been reported in several countries, such as the United Kingdom and New Zealand [1]. Most previous studies have rarely reported the isolation of $M$. bovis from rabbits. M. bovis was not isolated from 146 dead domestic rabbits in the United Kingdom between 1976-1979, and from about 1,200 dead wild rabbits in New Zealand in $1991[1,15]$. Based on the data by KAHIS, particularly, most cases were reported for cattle, and several cases have been reported in deer and otters in the Republic of Korea. As tuberculosis was not detected in domestic rabbits, they have been excluded from the list of target animals for national surveillance. This case suggests that rabbits need to be considered as a susceptible host and reservoir for M. bovis. In countries where bTB is prevalent, various potential hosts should be monitored for bTB infection.

\section{Acknowledgements}

This study was supported by a grant (Project code no. N1543069-2015-99-01) from the Animal and Plant Quarantine Agency (APQA), Ministry of Agriculture, Food and Rural Affairs (MAFRA), Republic of Korea.

\section{References}

1. Delahay RJ, De Leeuw AN, Barlow AM, Clifton-hadley RS, Cheeseman CL. The status of Mycobacterium bovis infection in UK wild mammals: a review. Vet J 2002;164:90-105.

2. Lee H, Kim JM, Jang Y, Lee K, Baek K, Lee B, Kim HY, Lee MH, Ryoo S, Bae YC, Choi EJ, So B. Bovine tuberculosis in an Asian small-clawed otter (Aonyx cinerea) in the Republic of Korea. J Vet Diagn Invest 2015;27:651655.

3. Verna AK, Tiwari R, Chakraborty S, Neha, Saminathan M, Dhama K, Singh SV. Insights into bovine tuberculosis (bTB), various approaches for its diagnosis, control and its public health concerns: an update. Asian J Anim Vet Adv 2014;9: 323-344.

4. Fitzgerald SD, Kaneene JB. Wildlife reservoirs of bovine tuberculosis worldwide: hosts, pathology, surveillance, and control. Vet Pathol 2013;50:488-499.

5. Jeong J, Lee K, Choi EJ, Kim HY, Sohn JH, So B, Jung JY. Meningoencephalitis and pneumonia caused by Pasteurella multocida in rabbits. Korean J Vet Res 2018;58:61-64.

6. Taylor GM, Worth DR, Palmer S, Jahans K, Hewinson RG. Rapid detection of Mycobacterium bovis DNA in cattle lymph nodes with visible lesions using PCR. BMC Vet Res 2007;3:12.

7. Yeboah-Manu D, Yates MD, Wilson SM. Application of a simple multiplex PCR to aid in routine work of the mycobacterium reference laboratory. J Clin Microbiol 2001; 39:4166-4168.

8. Jang Y, Ryoo S, Lee H, Kim N, Lee H, Park SY, Song WS, Kim JT, Lee HS, Kim JM. Isolation of Mycobacterium bovis from free-ranging wildlife in South Korea. J Wildl Dis 2017; 53:181-185.

9. Kim J, Kim JW, Oh SI, So B, Kim WI, Kim HY. Characterisation of Pasteurella multocida isolates from pigs with pneumonia in Korea. BMC Vet Res 2019;15:119.

10. Townsend KM, Frost AJ, Lee CW, Papadimitriou JM, Dawkins HJ. Development of PCR assays for species- and type-specific identification of Pasteurella multocida isolates. J Clin Microbiol 1998;36:1096-1100.

11. Kamerbeek J, Schouls L, Kolk A, van Agterveld M, van Soolingen D, Kuijper S, Bunschoten A, Molhuizen H, Shaw R, Goyal M, van Embden J. Simultaneous detection and strain differentiation of Mycobacterium tuberculosis for diagnosis and epidemiology. J Clin Microbiol 1997;35:907-914.

12. Smith $\mathrm{NH}$, Upton P. Naming spoligotype patterns for the RD9-deleted lineage of the Mycobacterium tuberculosis complex; www.Mbovis.org. Infect Genet Evol 2012;12:873876.

13. Barthold SW, Griffey SM, Percy DH. Rabbit. In: Barthold SW, Griffey SM, Percy DH (eds.). Pathology of laboratory rodents and rabbits. 4th ed. pp. 282. Wiley-Blackwell, New Jersey, 2016.

14. Calvete C, Estrada R, Villafuerte R, Osácar JJ, Lucientes J. Epidemiology of viral haemorrhagic disease and myxomatosis in a free-living population of wild rabbits. Vet Rec 2002; 150:776-782.

15. Allen GM. Other animals as sources of TB [tuberculosis] infection. In: Jackson R (ed.). Proceedings of a Symposium on Tuberculosis. New Zealand Veterinary Association; Foundation for Continuing Education, Palmerston North, pp. 197-201, 1991. 